\title{
Haloaldehyde Polymers. V. Polymer Blends Involving Chloral Polymers
}

\author{
L. S. Corley, P. Kubisa, and O. Vogl \\ Polymer Science and Engineering, University of Massachusetts, \\ Amherst, Massachusetts 01003 U.S.A.
}

(Received August 27, 1976)

\begin{abstract}
Blends and interpenetrating networks of polychloral with polystyrene, poly(methyl methacrylate), and poly(methyl acrylate) as addition polymers have been successfully prepared in the forms of cylinders (plugs) or of films of $0.03-\mathrm{mm}$ thickness by sequential polymerization. The addition polymers are completely extractable, but about $30 \%$ of the polychloral is degraded during the extraction. The polystyrene extracted from the polychloral-polystyrene blends was of relatively low molecular weight. This is attributed to the high chain transfer activity of chloral for styrene polymerization in the presence of chloral. Poly(methyl methacrylate) has a comparable molecular weight, as judged by its inherent viscosity, whether the polymer has been prepared in the presence or in the absence of chloral or polychloral. In the presence of crosslinking agents, divinylbenzene for styrene, and ethylene dimethacrylate for methyl methacrylate, interpenetrating networks of polychloral were obtained; it appears that about $30 \%$ of the olefinic polymer (polystyrene) is not crosslinked and can be extracted. Poly(methyl methacrylate) is completely crosslinked. TGA was found to be a simple technique to analyze the composition of the polymer blends.

KEY WORDS Polychloral / Polystyrene / Poly(methyl methacrylate) / Poly(methyl acrylate) / Polymer Blends / Interpenetrating Polymer Networks / Triphenylphosphine / Azobisisobutyronitrile / Thermal Degradation / Solvent Extraction / Degradative Extraction /
\end{abstract}

Chloral homo- and copolymers with high chloral content are infusible and insoluble in all solvents ${ }^{1}$ and consequently cannot be blended with other polymers by conventional melt or solution blending techniques.

Chloral polymers must be prepared by cryotachensic polymerization, a monomer casting technique which involves initiation above the polymerization temperature and subsequent cooling of the mixture in order to cause polymerization to a homogeneous gel. ${ }^{2}$ Special procedures have been devised for the preparation of blends of chloral polymers with other polymers. Three basic techniques have been described ${ }^{3}$ as successful for the preparation of blends of chloral polymers with addition polymers: (a) dissolving the addition polymer in chloral, adding initiator to chloral above the polymerization threshold temperature, and then cooling to polymerize chloral; (b) imbibing the addition monomer (which contains the initiator) into polychloral pieces and then polymerizing the addition monomer within the polychloral matrix; and (c) preparing a mixture of chloral, the second monomer, and a radical initiator for the olefinic monomer and heating the mixture above the threshold temperature. The initiator for the chloral polymerization is then added and the polymerization is carried out by sequential polymerization, whereby chloral is first polymerized by cooling followed by the polymerization of one (or a mixture of more than one) addition monomers.

Examples of all these techniques have been described in the literature. ${ }^{3}$ For the preparation of polymer blends by dissolving the addition polymer in chloral or in a mixture of chloral and isocyanate, a convenient polymerization mixture, blends were obtained of addition 


\section{S. Corley, P. Kubisa, and O. Vogl}

polymers with chloral polymers or copolymers. Examples have been given for "apparently homogeneous" blends which contained ethylene/propylene rubber (4\%), polystyrene $(8.5 \%)$, polyacrylates (7\%), poly(methacrylate)s (7-9\%), and poly( $N$-vinylcarbazole) $(9 \%)$. The limitations for this polymerization technique were that (a) the added polymer must remain soluble in the polymerizing mixture of chloral, particularly at the end of the polymerization, and (b) the polymer must not interfere with the chloral polymerization. As a consequence, it must not have active hydrogen groups or groups that would interfere in this anionic chloral polymerization. Polyblends incorporating rubbery polymers showed a five-fold improvement in impact strength over unmodified chloral polymers.

The second technique used to prepare blends of chloral polymers with addition polymers involved preparing polychloral pieces in their final form, imbibing the polychloral pieces with an addition monomer, and then polymerizing the addition monomer by conventional means. This technique of preparing polyblends involving polychloral also has its limitations, principally the capacity of the chloral polymer to imbibe the addition monomer, but polyblends with as much as $55 \%$ of addition monomer in the polychloral matrix were prepared. It has not been demonstrated that these monomers actually swelled polychloral. The addition monomers filled voids in the polychloral pieces but substantial differences in reproducibility and differences in the amounts of individual monomers incorporated into the polychloral matrix were not explained. ${ }^{3}$ It is, however, clear that the monomers used in the preparation of these blends were substantially different in polarity and reactivity. One advantage of this technique was that radical, cationic, and anionic polymerization techniques could be used for the polymerization of addition monomers.

Poly(methyl methacrylate) could be incorporated in chloral polymers to $28 \%$, poly(methyl acrylate) to $28 \%$, poly(methacrylic acid) to $10 \%$, poly(butyl methacrylate) to $13 \%$, poly(2-ethylhexyl methacrylate) to $10 \%$, and polyacrylonitrile to $18 \%$. Aromatic vinyl polymers were also successfully incorporated: polystyrene to $29 \%$, $\operatorname{poly}(\alpha$-methylstyrene) to $6 \%$, and poly $(N$-vinyl- carbazole) to $11 \%$. The preparation of these types of blends was carried out by soaking the polychloral pieces in the addition monomer which also contained a radical initiator, usually AIBN. The polymerization of the addition monomer was then carried out by heating to decompose the radical initiator. Polyisoprene was incorporated to $16 \%$ and poly(2,3-dichlorobutadiene) to $9 \%$. An unusual result was obtained with polychloroprene, which was incorporated to $55 \%$ and gave a leathery polymer. This particular experiment apparently caused some grafting of the chloroprene onto the polychloral chains.

Other monomers were polymerized in the polychloral matrix by ionic initiators. $\operatorname{Poly}(\beta-$ propiolactone) was incorporated to $7 \%$ with tributyl phosphine as the initiator. Poly(propylene oxide) could be incorporated to $24 \%$, poly(epichlorohydrin) to $31 \%$, and poly(2-ethylhexyl vinyl ether) to $11 \%$, when the polychloral sample was soaked with the monomer and the polymerization was carried out by inserting the imbibed polychloral piece into an atmosphere which contained $\mathrm{BF}_{3}$ diethyl etherate.

In many cases of the incorporation of an addition polymer into the polychloral matrix, the mechanical properties of the polychloral were substantially altered. A typical sample of polychloral with a flexural strength of $70 \mathrm{MPa}$ and an elongation of several percent had a flexural modulus of $2400 \mathrm{MPa}$ and an Izod impact strength of about 0.5 . The flexural strength of the modified polymers increased from 40 to 80 $\mathrm{MPa}$, the Izod impact strength from 0.4 to 0.7 , the flexural modulus from 1000 to $2400 \mathrm{MPa}$, and the elongation to $15 \%$.

A third and potentially most versatile method for the preparation of composites of chloral polymers with addition polymers is the method of sequential polymerization. It was found earlier that this method could be most conveniently carried out in the following way. Chloral and its comonomer, for example, phenyl isocyanate, were mixed with an addition monomer and a radical initiator and the mixture was heated above the polymerization temperature of chloral. The initiator for the chloral polymerization was then added and the chloral was polymerized by cryotachensic polymerization. After 
the chloral polymerization was completed, the mixture was heated to the temperature needed to activate the radical initiator and the radical polymerization was then carried out.

Conversions of chloral to polychloral of more than $96 \%$ have been reported in the presence of $20 \%$ of an inert diluent, although conversions in bulk polymerizations do not exceed $85 \% .^{4,5}$ The use of highly volatile diluents for chloral polymerization has been employed for the preparation of foams and sponges of chloral polymers. ${ }^{6}$ Most addition monomers, particularly olefinic monomers such as styrene, $\alpha$-methylstyrene, and also methyl methacrylate, are inert diluents for the chloral polymerization. The limitations on the use of individual monomers are the volatility of the monomers and their reactivity, or that of the growing polymeric radical or alkoxide, with the remaining chloral monomer. The use of addition monomers as "inert diluents" in this sequential polymerization involving chloral has the advantage that initially much higher conversions can be obtained in the chloral polymerization as compared to its bulk polymerization, and, after the addition monomer has been polymerized, a composite blend is produced which is more uniform and void-free than polychloral homo- or copolymer samples of predetermined final structure, such as a film or a sheet.

Chloral homopolymers and copolymers with more than $80 \mathrm{~mol} \%$ of chloral units are infusible and insoluble and behave as if they were crosslinked. It was, therefore, considered possible to prepare interpenetrating networks containing two continuous phases, the polychloral phase and the phase of a crosslinked olefinic polymer, by adding a crosslinking agent to the olefinic monomer.

Interpenetrating polymer networks are commonly prepared $^{7}$ by swelling a crosslinked polymer, for example, a polyurethane, with a second monomer containing a multifunctional comonomer as a crosslinking agent. A typical example is an acrylate or methacrylate (with a bisacrylate or bismethacrylate as a crosslinking agent) and an initiator for the polymerization of the acrylic monomer. The second monomer (and comonomer as crosslinking agent) are then polymerized within the matrix of the first lightly crosslinked polymer (the polyurethane), to produce a crosslinked network within the first polymer network.

In our early work ${ }^{3}$ on the preparation of blends of chloral polymers with addition polymers, a number of monomers were studied for sequential polymerization, most prominently styrene, methyl methacrylate, and methyl acrylate. It could be shown that the addition polymers could be extracted from the polymer blends to an extent of more than $95 \%$. Consequently, no grafting of the addition monomer to the polychloral portion had occurred under these conditions.

It is remarkable that it was possible to initiate successfully two polymerizations in sequence by two different mechanims without substantial interference of each of the polymerizations with the other.

The objective of this work is to prepare, by sequential polymerization techniques, a series of blends and interpenetrating networks of chloral homopolymer with polystyrene, poly(methyl methacrylate), and poly(methyl acrylate).

\section{EXPERIMENTAL}

\section{Materials}

Chloral (Montrose Chemical Co.) was dried over $\mathrm{P}_{2} \mathrm{O}_{5}$, distilled, ${ }^{8}$ and used immediately.

Styrene (Eastman Kodak Co.) was dried over calcium hydride for $24 \mathrm{hr}$ and distilled under reduced pressure; bp $33-35^{\circ} \mathrm{C}(8 \mathrm{~mm})$.

Methyl methacrylate (MMA) (Eastman Kodak Co.) was dried over calcium hydride and distilled at atmospheric pressure; bp $100-101^{\circ} \mathrm{C}$.

Methyl acrylate (MA) (Aldrich Chemical Co.) was dried over calcium hydride and distilled at atmospheric pressure; bp $80-81^{\circ} \mathrm{C}$.

Divinylbenzene (DVB) (MC/B Manufacturing Chemists) was dried and distilled under reduced pressure; bp $52-53^{\circ} \mathrm{C}(4 \mathrm{~mm})$. Gas chromatography showed the distillate to consist of $60-\%$ divinylbenzenes and 40-\% vinylethylbenzene, with a trace amount of diethylbenzenes of various isomer compositions.

Ethylene dimethacrylate (EDMA) (Pfaltz, Bauer Inc.) was dried with $\mathrm{CaH}_{2}$ and distilled under reduced pressure; bp $85-86^{\circ} \mathrm{C}(2 \mathrm{~mm})$.

Triphenylphosphine $\left(\mathrm{Ph}_{3} \mathrm{P}\right)$ (Aldrich Chemical 


\section{S. Corley, P. Kubisa, and O. Vogl}

Co.) was recrystallized from methanol and used as a $1-M$ solution in benzene.

Azobisisobutyronitrile (AIBN) (Eastman Kodak Co.) was recrystallized from methanol and used as a $0.3-M$ solution in benzene.

Benzene (Fisher Scientific Co., Certified A.C.S. Reagent Grade) was dried and stored over sodium wire.

Methanol, acetone, toluene, and dichloromethane were reagent grade solvents obtained from the Fisher Scientific Co. and were used without purification.

Glass plates for the film preparation assembly were Pyrex, obtained from the SGA Scientific Co.

Rubber thread for the assembly was Lycra, obtained from the Globe Manufacturing Co., Fall River, Mass.

\section{Polymer Preparation}

A typical preparation of a blend of polychloral with polystyrene by sequential polymerization is as follows: Into a flamed-out serum-stoppered $15 \mathrm{~mm} \times 125 \mathrm{~mm}$ test tube were injected (with syringes) $4 \mathrm{ml}(6.05 \mathrm{~g}, 41 \mathrm{mmol})$ of freshly distilled chloral, $1.89 \mathrm{ml}(1.71 \mathrm{~g}, 16.4 \mathrm{mmol})$ of styrene, and $0.16 \mathrm{ml}(0.048 \mathrm{mmol})$ of a $0.3-M$ solution of AIBN in benzene. The tube was placed into an oil bath at $70^{\circ} \mathrm{C}$. After $5 \mathrm{~min}$ the tube contents had reached bath temperature. Then, $0.33 \mathrm{mmol}$ of $\mathrm{Ph}_{3} \mathrm{P}$ was added as a $1-M$ solution in benzene and the tube was shaken to mix the reactants. The clear solution was then rapidly transferred with a hot syringe to a hot assembly ${ }^{8}$ to prepare a polychloral film. The assembly was then placed into an icewater bath for $16 \mathrm{hr}$. It was taken out, allowed to air dry, and placed into an oven at $70^{\circ} \mathrm{C}$. After one day the glass plates were separated and each polymer film was trimmed and cut into two pieces. The first part was dried for $24 \mathrm{hr}$ at room temperature at $0.02 \mathrm{mmHg}$. The second piece was extracted with methanol (to remove unreacted monomers) in a test tube for 4 days at room temperature, and dried for one day at $0.02 \mathrm{mmHg}$. The methanol extracted film was then divided into two pieces, one of which was extracted with dichloromethane to remove polystyrene.

The polymer cylinder from the test tube was similarly divided into three pieces for extraction. Weights of the individual cylinders were deter- mined before and after vacuum drying or solvent extraction for each cylinder. Respective total conversions for each monomer (from elemental analysis or thermogravimetric measurements on the extracted cylinders) were calculated for each sample. The "conversions" calculated for the individual monomers to polymers for cylinders extracted with each solvent referred strictly to the amounts of each polymer remaining in the sample after extraction. The amount of each monomer used is considered $100 \%$.

The polymer samples consisting of the blends of polychloral and polystyrene were first extracted with acetone at room temperature for 4 days. In some cases the extraction removed as much as $50-60 \%$ of polystyrene of low molecular weight. In addition, absorbed chloral monomer was also extracted and the unstable polychloral portion was degraded to chloral monomer. As a consequence, the remaining polymer sample consisted of stable polychloral and polystyrene of sufficient molecular weight to be not soluble in, or extractable by, acetone.

The samples were then dried for $24 \mathrm{hr}$ at room temperature at $0.02 \mathrm{mmHg}$ and then extracted with $\mathrm{CH}_{2} \mathrm{Cl}_{2}$ for 4 days at room temperature (4 changes of $\mathrm{CH}_{2} \mathrm{Cl}_{2}(40 \mathrm{ml})$ for $1.5-\mathrm{g}$ sample of polymer). The combined extracts were evaporated for $24 \mathrm{hr}$ at atmospheric pressure and room temperature. The residues of the extracts were weighed and the conversions of addition monomers to extractable polymers were calculated. The extracts $(0.1-0.5 \mathrm{~g})$ were then redissolved in $15-\mathrm{ml}$ of dichloromethane, reprecipitated from $350 \mathrm{~m} l$ of methanol filtered, and dried; and the samples were characterized by their PMR spectra and by measurement of the solution viscosity.

The polychloral blends with linear poly(methyl methacrylate) and poly(methyl acrylate) were extracted and characterized in the same manner as the polystyrene blends, except that the blends with poly(methyl acrylate) were not extracted with dichloromethane.

The interpenetrating networks of polychloral with crosslinked polystyrene and crosslinked poly(methyl methacrylate) were prepared only in film form and were extracted with refluxing benzene alone. 
Haloaldehyde Polymers. V.

\section{Measurements}

Thermogravimetric measurements (TGA) were performed on shavings from the extracted polymer samples with a Perkin-Elmer TGS-1 Thermobalance from ambient temperature to $430^{\circ} \mathrm{C}$ at a heating rate of $10^{\circ} \mathrm{C} / \mathrm{min}$. For each set of blends, it was found that the polychloral degraded essentially completely before the addition polymer began to degrade. The percentage of total weight loss above a specified temperature $\left(327^{\circ} \mathrm{C}\right.$ for polystyrene, $302^{\circ} \mathrm{C}$ for poly(methyl acrylate), and $277^{\circ} \mathrm{C}$ for poly(methyl methacrylate)) could be plotted linearly as a function of olefinic addition polymer content of the blends as determined by carbon-hydrogen analysis (Figure 1).

Infrared spectra of the films were recorded on a Perkin-Elmer Model 727 Infrared Spectrophotometer.

PMR spectra of $\mathrm{CDCl}_{3}$ solutions of extracted addition polymers were recorded on a Hitachi Perkin-Elmer R-24 60-MHz NMR Spectrometer.

Inherent viscosities of polymers extracted from the blends by solvents were determined in toluene at $30^{\circ} \pm 0.3^{\circ} \mathrm{C}$ at a concentration of $0.5 \mathrm{~g} / \mathrm{d} l$ with a Ubbelohde Viscometer.

Elemental analyses were performed by the Microanalysis Laboratory of the University of Massachusetts.

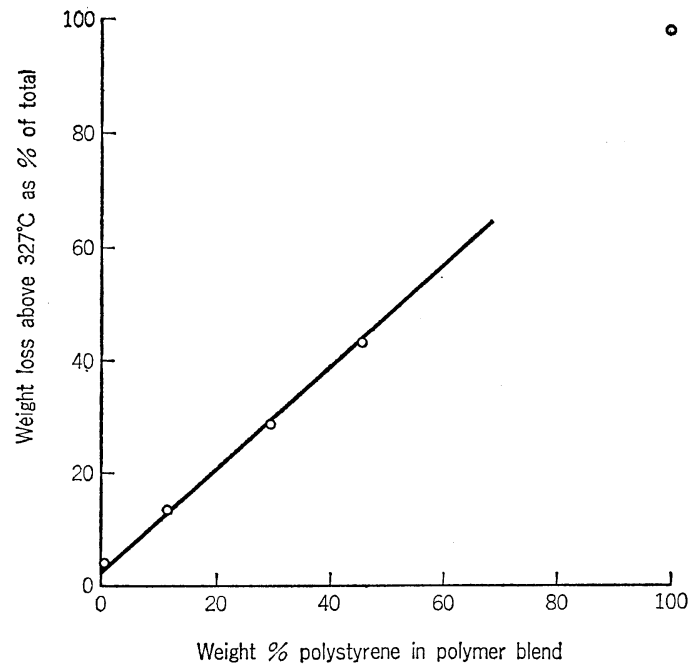

Figure 1. Thermal degradation of blends of polychloral with polystyrene: Heating rate, $10^{\circ} \mathrm{C} / \mathrm{min}$; sample size, $2 \mathrm{mg}$.

\section{RESULTS AND DISCUSSION}

Polymer blends and interpenetrating networks of chloral homopolymers with various olefinic addition polymers have been prepared by a sequence of two polymerizations. Initially the mixture of chloral, the olefinic addition monomer, and its initiator was heated above the threshold temperature of chloral polymerization. The initiator for chloral polymerization was then added and the chloral was polymerized by cryotachensic polymerization. After the chloral polymerization the mixture was warmed to the decomposition temperature of the radical initiator for the polymerization of the olefinic addition monomer and this polymerization was then carried out within the polychloral matrix.

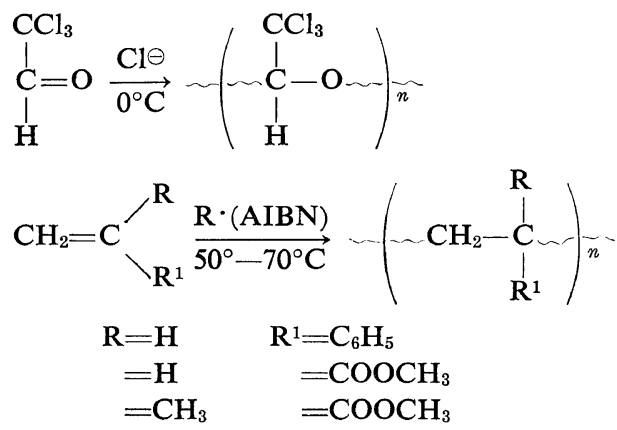

In order to evaluate the successful preparation of the blends and interpenetrating networks, the samples were usually divided into three pieces. One portion was held overnight at room temperature and $0.025 \mathrm{mmHg}$ to remove volatile monomers and solvent. The second part was extracted with methanol. The third portion was extracted with benzene, acetone, or methylene dichloride. The individual samples were then analyzed for their composition by elemental analysis, TGA, and IR; the results are recorded in Tables I-V.

It has been established that blends and interpenetrating networks of polychloral with polystyrene, poly(methyl methacrylate) (PMMA), and poly(methyl acrylate) (PMA) could be prepared in compositions near the initial feed compositions by this sequential polymerization technique. Polymer blends of polychloral with polystyrene, PMMA, and PMA were formed as translucent plugs or cylinders when the polymerization was carried out directly in the test 
Table I. Composition of polychloral-polystyrene blends ${ }^{\mathrm{a}}$

\begin{tabular}{|c|c|c|c|c|c|c|c|}
\hline \multirow[b]{2}{*}{$\begin{array}{c}\text { Styrene, } \mathrm{b} \\
\text { mol } \% \\
(\text { wt } \%)\end{array}$} & \multirow[b]{2}{*}{$\begin{array}{l}\mathrm{Ph}_{3} \mathrm{P}, \mathrm{c} \\
\mathrm{mol} \%\end{array}$} & \multirow{2}{*}{$\begin{array}{l}\text { Total } \\
\text { conversion } \\
\text { after } \\
\text { vacuum } \\
\text { drying, } \\
\text { wt } \%\end{array}$} & \multicolumn{4}{|c|}{ After methanol extraction } & \multirow{2}{*}{$\begin{array}{c}\text { Chloral } \\
\text { conversiond } \\
\text { after } \mathrm{CH}_{2} \mathrm{Cl}_{2} \\
\text { extraction, } \\
\% \\
\%\end{array}$} \\
\hline & & & $\begin{array}{c}\text { Total } \\
\text { conversion, } \\
\%\end{array}$ & $\begin{array}{c}\text { Chloral } \\
\text { conversion, } \\
\%\end{array}$ & $\begin{array}{c}\text { Styrene } \\
\text { conversion, } \\
\%\end{array}$ & $\begin{array}{c}\text { Polychloral- } \\
\text { polystyrene } \\
\text { ratio } \\
\text { by weight }\end{array}$ & \\
\hline 0 & 0.2 & 87 & 68 & 68 & - & 100 & 72 \\
\hline $17 \quad(12.7)$ & 0.4 & 88 & 65 & 66 & 60 & $89: 11$ & 55 \\
\hline $28.5(22.0)$ & 0.8 & 95 & 74 & $73^{e}$ & $79^{e}$ & $77: 25$ & 64 \\
\hline $37.5(29.8)$ & 1.2 & 94 & 66 & 66 & 67 & $70: 30$ & 52 \\
\hline $44.5(36.2)$ & 1.6 & 95 & 67 & $62^{\circ}$ & $75^{\circ}$ & $59: 41$ & 51 \\
\hline $50 \quad(41.5)$ & 2.0 & 93 & 63 & 58 & 70 & $54: 46$ & 47 \\
\hline
\end{tabular}

a Monomer cast in cylinders (plugs); $0.3-\mathrm{mol} \%$ AIBN with respect to olefinic addition monomer used as initiator.

b Total number of moles of both monomers in mixture is 100 .

c Chloral in initial monomer mixture is 100 .

d Conversion to polymer after extraction.

e Determined by TGA; other compositions determined by elemental analysis $(\mathrm{C}, \mathrm{H})$.

\& Quantitative extraction of polystyrene.

Table II. Composition of polychloral-poly(methyl methacrylate) blends ${ }^{a}$

\begin{tabular}{|c|c|c|c|c|c|c|c|}
\hline \multirow[b]{2}{*}{$\begin{array}{l}\text { MMA, } \\
\text { mol \% } \\
(\text { wt } \%)\end{array}$} & \multirow[b]{2}{*}{$\begin{array}{l}\mathrm{Ph}_{3} \mathrm{P},{ }^{\mathrm{c}} \\
\mathrm{mol} \%\end{array}$} & \multirow{2}{*}{$\begin{array}{l}\text { Total } \\
\text { conversion } \\
\text { after } \\
\text { vacuum } \\
\text { drying, } \\
\text { wt } \%\end{array}$} & \multicolumn{4}{|c|}{ After methanol extraction } & \multirow{2}{*}{$\begin{array}{c}\text { Chloral } \\
\text { conversiond } \\
\text { after } \mathrm{CH}_{2} \mathrm{Cl}_{2} \\
\text { extraction, } \\
\%\end{array}$} \\
\hline & & & $\begin{array}{c}\text { Total } \\
\text { conversion, }{ }^{d} \\
\%\end{array}$ & $\begin{array}{c}\text { Chloral } \\
\text { conversion, } \\
\%\end{array}$ & $\begin{array}{c}\text { MMA } \\
\text { conversion, } \\
\%\end{array}$ & $\begin{array}{l}\text { Polychloral- } \\
\text { PMMA } \\
\text { ratio in } \\
\text { final polymer }\end{array}$ & \\
\hline 0 & 0.2 & 87 & 68 & 68 & - & 100 & 72 \\
\hline $17 \quad(12)$ & 0.4 & 100 & 98 & 93 & 100 & $83: 17$ & 99 \\
\hline $28.5(21)$ & 0.8 & 100 & 88 & $82^{\mathrm{e}}$ & $100^{\circ}$ & $67: 33$ & 100 \\
\hline $37.5(29)$ & 1.2 & 100 & 88 & 77 & 100 & $63: 37$ & 82 \\
\hline $44.5(35)$ & 1.6 & 100 & 94 & $91^{\mathrm{e}}$ & $98^{\circ}$ & $63: 37$ & 81 \\
\hline $50 \quad(40)$ & 1.8 & 100 & 84 & 70 & 100 & $51: 49$ & 50 \\
\hline
\end{tabular}

a Monomer cast in cylinders (plugs); $0.3-\mathrm{mol} \%$ AIBN used as initiator (with respect to olefinic addition monomer).

b Total numbers of both monomers in mixture is 100 .

c Chloral in initial monomer mixture is 100 .

d Conversion of polymer after extraction.

- Determined by TGA; other compositions determined by elemental analysis (C, H).

tube or as transparent films when the polymerization was carried out in the assembly for polymer film preparation. Plugs and films could be extracted with solvents for the olefinic addition polymers or with nonsolvents for these polymers. If nonsolvents were used, for example methanol, only monomers and very low molecular weight polymers were extracted. When good solvents, such as acetone, benzene, or methylene dichloride, were used, the addition polymer was either completely extracted or extracted to an extent of more than $95 \%$, which indicated that no grafting of the addition polymer on the polychloral was obtained. It has, however, been shown that unreacted chloral acts as a chain transfer agent in the polymerization of the olefinic addition polymer, even though the initiator used for these polymerizations, AIBN, produces radicals which are not sufficiently reactive to abstract chlorine from the polychloral matrix, thus causing no grafting. Furthermore, the growing polymeric radicals 
Haloaldehyde Polymers. V.

Table III. Composition of polychloral-poly(methyl acrylate) blends ${ }^{a}$

\begin{tabular}{|c|c|c|c|c|c|c|c|}
\hline \multirow[b]{2}{*}{$\begin{array}{l}\mathrm{MA}, \mathrm{b} \\
\mathrm{mol} \% \\
(\mathrm{wt} \%)\end{array}$} & \multirow[b]{2}{*}{$\begin{array}{l}\mathrm{Ph}_{3} \mathrm{P}, \mathrm{c} \\
\mathrm{mol} \%\end{array}$} & \multirow{2}{*}{$\begin{array}{c}\text { Total } \\
\text { conversion } \\
\text { after } \\
\text { vacuum } \\
\text { drying, } \\
\text { wt } \%\end{array}$} & \multicolumn{4}{|c|}{ After methanol extraction } & \multirow[b]{2}{*}{$\begin{array}{c}\text { Chloral } \\
\text { conversion }^{\mathrm{d}} \\
\text { after acetone } \\
\text { extraction, } \\
\%\end{array}$} \\
\hline & & & $\underset{\substack{\text { Total } \\
\text { conversion, } \\
\%}}{\mathrm{~d}}$ & $\begin{array}{c}\text { Chloral } \\
\text { conversion, } \\
\%\end{array}$ & 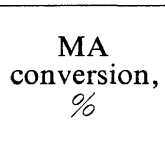 & $\begin{array}{l}\text { Polychloral- } \\
\text { PMA } \\
\text { ration in } \\
\text { final product }\end{array}$ & \\
\hline 0 & 0.2 & 88 & 68 & 68 & - & 100 & 72 \\
\hline $17 \quad(10.6)$ & 0.4 & 100 & 83 & 79 & 100 & $86: 14$ & 84 \\
\hline $28.5(18.9)$ & 0.8 & 95 & 64 & $58^{\circ}$ & $92^{\ominus}$ & $73: 27$ & 47 \\
\hline $37.5(26.0)$ & 1.2 & 96 & 57 & 45 & 90 & $59: 41$ & 39 \\
\hline $44.5(32.1)$ & 1.6 & 89 & 50 & $26^{\circ}$ & $99^{\circ}$ & $36: 64$ & 46 \\
\hline $50 \quad(36.9)$ & 2.0 & 86 & 44 & 24 & 78 & $35: 65$ & 32 \\
\hline
\end{tabular}

a Monomer cast in cylinders (plugs); 0.3-mol \% AIBN with respect to olefin addition monomer used as initiator.

b Total number of moles of both monomers in mixture is 100 .

c Chloral in intial monomer mixture is 100 .

d Conversion to polymer after extraction.

- Determined by TGA; other compositions determined by elemental analysis $(\mathrm{C}, \mathrm{H})$.

Table IV. Composition of polychloral-crosslinked polystyrene interpenetrating networks ${ }^{\mathrm{a}}$

\begin{tabular}{|c|c|c|c|c|c|}
\hline \multirow[b]{2}{*}{$\begin{array}{c}\text { Styrene,b } \\
\text { mol } \% \\
(\text { wt } \%)\end{array}$} & \multirow[b]{2}{*}{$\begin{array}{l}\operatorname{mol} \% \\
\mathrm{Ph}_{3} \mathrm{P}, \mathrm{c}\end{array}$} & \multirow[b]{2}{*}{$\begin{array}{c}\text { AIBN,d } \\
\text { mol } \%\end{array}$} & \multirow[b]{2}{*}{$\begin{array}{c}\text { Total } \\
\text { conversion } \\
\text { after vacuum } \\
\text { drying, } \\
\text { wt } \%\end{array}$} & \multicolumn{2}{|c|}{ After benzene extraction } \\
\hline & & & & $\begin{array}{c}\text { Total } \\
\text { conversion, } \\
\text { wt } \%\end{array}$ & $\begin{array}{c}\text { Polychloral- } \\
\text { crosslinked } \\
\text { polystyrene ratio } \\
\text { in final polymer } \\
\text { by weight }\end{array}$ \\
\hline $17.5(13)$ & 0.25 & 0.66 & 92 & 55 & $95: 5$ \\
\hline $36 \quad(29)$ & 0.33 & 0.66 & 94 & 42 & $84: 16$ \\
\hline $56 \quad(47)$ & 0.50 & 0.60 & 91 & 49 & $73: 27$ \\
\hline
\end{tabular}

a Monomer cast in films, $\sim 0.03 \mathrm{~mm}$ thick.

b Contains 5.4-\% divinylbenzene (with respect to total monomer content).

c Chloral is 100 .

d Styrene/divinylbenzene mixture is 100 .

e Based on weight of film directly after polymerization, not on weight of original monomer mixture.

Table V. Composition of polychloral-crosslinked poly(methyl methacrylate) interpenetrating networks ${ }^{a}$

\begin{tabular}{|c|c|c|c|c|c|}
\hline \multirow[b]{2}{*}{$\begin{array}{l}\text { MMA, } \\
\text { mol \% } \\
(w t \%)\end{array}$} & \multirow[b]{2}{*}{$\begin{array}{l}\mathrm{Ph}_{3} \mathrm{P}, \mathrm{c} \\
\mathrm{mol} \%\end{array}$} & \multirow[b]{2}{*}{$\begin{array}{l}\text { AIBN,d } \\
\text { mol } \%\end{array}$} & \multirow[b]{2}{*}{$\begin{array}{c}\text { Total } \\
\text { conversion } \\
\text { after vacuum } \\
\text { drying, } \\
\text { wt } \%{ }^{\ominus}\end{array}$} & \multicolumn{2}{|c|}{ After benzene extraction } \\
\hline & & & & $\begin{array}{c}\text { Total } \\
\text { conversion, } \\
\text { wt } \%{ }^{\mathrm{e}}\end{array}$ & $\begin{array}{l}\text { Polychloral- } \\
\text { crosslinked } \\
\text { PMMA ratio } \\
\text { in final polymer } \\
\text { by weight }\end{array}$ \\
\hline $19 \quad(14)$ & 0.37 & 1.0 & 95 & 64 & $97: 3$ \\
\hline $39 \quad(30)$ & 0.50 & 0.9 & 98 & 60 & $54: 46$ \\
\hline$(48)$ & 0.75 & 1.2 & 98 & 74 & $32: 68$ \\
\hline
\end{tabular}

a Monomer cast in films, $0.03 \mathrm{~mm}$ thick.

b Contains 7.6-\% ethylene dimethacrylate (EDMA) (with respect to total monomer content).

c Chloral is 100 .

d MMA/EDMA mixture is 100 .

- Based on weight of film directly after polymerization, not on weight of original monomer mixture. 
produced by the addition monomers used in this investigation are also apparently not reactive enough to abstract the chlorine from the polychloral matrix.

As in other anionic cryotachensic polymerizations, polychloral is deposited as a gel from the polymerization mixture; this gel appears homogeneous, but its detailed structure and morphology have not been investigated thoroughly. However, it has chloral monomer, solvent, and, in the case of sequential polymerization, the addition monomer imbibed within its structure. The polychloral gel, however, is insoluble and most probably this insolubility is a contributing factor in its unreactivity in the subsequent radical polymerization of the addition monomer.

All our evidence has indicated that chloral polymerizes to a polymer which consists of a stable polychloral fraction, an unstable polychloral fraction, and unreacted chloral monomer which is tightly associated with the polychloral sample. Evaporation at $0.025 \mathrm{mmHg}$ at room temperature overnight removed only a substantial part of the unreacted monomer. The remainder is apparently largely used up during the polymerizatiou of the addition monomer as a chain transfer agent. A substantial portion of the chloral polymer prepared with $\mathrm{Ph}_{3} \mathrm{P}$ was unstable and was degraded to chloral monomer which was leached out by the solvent when the polymer sample was extracted with methanol, acetone, or methylene chloride. In the case of methanol extraction the chloral is extracted as the hemiacetal.

The polymerization of the olefinic addition monomers styrene, MMA, and MA apparently goes to completion with AIBN, the initiator which was exclusively used for this polymerization. Other radical initiators such as benzoyl peroxide or other acyl peroxides or hydroperoxides were not considered because it was expected that they would interfere with the chloral polymerization. Other peroxides, such as tertiary butyl peroxide, could not be used because their decomposition temperature was too high, within the range where thermal depolymerization of polychloral, which had been formed in the first part of the sequential polymerization, occurred at a significant rate.

\section{POLYMER BLENDS}

\section{Polychloral-Polystyrene Blends}

Blends of polychloral with polystyrene were prepared primarily in the form of $12 \times 50-\mathrm{mm}$ plugs in test tubes with $\mathrm{Ph}_{3} \mathrm{P}$ as initiator for the chloral and AIBN for the styrene polymerization. Blends were prepared of up to 50-mol\% styrene in the monomer feed. It was found in initial experiments that an increase in $\mathrm{Ph}_{3} \mathrm{P}$ initiator concentration was required to produce satisfactory chloral polymerization with decreasing chloral concentration in the mixture. As a consequence, as the concentration of styrene monomer in the mixture was increased from 0 to $50 \mathrm{~mol} \%$, the amount of $\mathrm{Ph}_{3} \mathrm{P}$ used to initiate the chloral polymerization was also increased from 0.2 to $2.0 \mathrm{~mol} \%$. The initiator concentration for the styrene polymerization was $0.3-\mathrm{mol} \%$ AIBN in all cases. This particular balance, which is described specifically in Table I, was found appropriate to prepare good blends of polychloral and polystyrene. After the polymerization was completed, the polymer blends were freed of volatiles by overnight drying at $0.025 \mathrm{mmHg}$ at room temperature, after which treatment $87-93 \%$ of polymer was left. Other portions of the polymer sample were also extracted with methanol, which is a nonsolvent for high molecular weight polystyrene and of course also a nonsolvent for polychloral. After four days of soaking with daily changes of solvent, $65-74 \%$ of the polymer blend remained, depending on the individual blend composition.

As indicated earlier, this treatment removed unreacted chloral if there was any left and degraded unstable, most probably living but dormant, polychloral terminated with alkoxide end groups. The chloral conversion was 58$73 \%$, as determined by carbon/hydrogen analysis or, more simply and equally effectively, by determination of the fraction of the blend degrading above $327^{\circ} \mathrm{C}$ under TGA conditions at a heating rate of $10^{\circ} \mathrm{C} / \mathrm{min}$, which was linearly related to the fraction of polystyrene in the blend (Figure 1). Below this temperature, polychloral degrades essentially quantitatively to chloral monomer, which evaporates, and polystyrene does not degrade at all. This temperature and these conditions have been carefully worked out in test 
experiments, the results of which are summarized in Figure 1.

The methanol treatment left about 60-80\% of the polystyrene in the polymer blend, as determined by elemental analysis and/or by the TGA degration of the polymer composite. Polystyrene of very low molecular weight and some small amount of styrene monomer were extracted by the methanol. The methanol solution left a gummy residue upon evaporation.

The final compositions of the polychloralpolystyrene blends after methanol extraction showed that a polymer blend which was prepared from a chloral-styrene mixture with a weight fraction of $12.7 \%$ of styrene had $11 \%$ of polystyrene in the blend. A polychloralpolystyrene blend which contained $25 \%$ of polystyrene after methanol extraction was obtained from a monomer mixture with $22 \%$ of styrene in the feed. A polychloral-polystyrene blend with $30 \%$ of polystyrene was produced with an almost similar feed ratio. As the ratio of styrene to chloral in the monomer feed increased, the polychloral-polystyrene blends became slightly richer in polystyrene. This seems to indicate that the polymerization of chloral is somewhat decreased at high styrene concentration or that the proportion of unstable polychloral in the total increases as the polychloral-polystyrene blend increases in polystyrene composition. This is also borne out by the observation that although total conversion after vacuum drying remains relatively constant with increasing styrene/chloral ratio, the total percentage of chloral conversion to polychloral after methanol extraction decreases slightly.

If one compares the observed apparent conversion of chloral after methylene chloride extraction with the styrene content of the monomer feed, one finds that the percentage of stable polychloral decreased as the styrene content increased in the original monomer mixture from $72 \%$ for no styrene to $47 \%$ for 41.5 -wt $\%$ styrene.

There could be three reasons for this result: a higher fraction of dormant living polychloral, a greater surface area of polychloral in the blend which is exposed during extraction, or lower molecular weight polychloral. We expect that all three contributions play a role in the instability of such polychloral samples, but in our experience, a larger surface area of the sample and better penetration of the degrading solvent into the sample are probably the most important factors. In a separate paper on perhaloaldehyde we will point out that polychloral initiated with $\mathrm{Ph}_{3} \mathrm{P}$ exists exclusively with polymer with low thermal stability. The maximum rate of degradation (MDT) of the polymer samples are between $120^{\circ} \mathrm{C}$ and $140^{\circ} \mathrm{C}$, cationically prepared polymer samples have a MDT of $250^{\circ} \mathrm{C}$ and the best stabilized polychloral samples degrade at $380^{\circ} \mathrm{C}$. Since polychloral does not swell substantially in any solvent, the size of the surface of the polymer is probably primarily responsible for the variations of the stable fractions under apparently very similar conditions.

Polystyrene was isolated from the polychloralpolystyrene blends by an initial acetone extraction, which was followed by a methylene chloride extraction. Polystyrene obtained from these blends by methylene chloride extraction of samples previously extracted by acetone had an inherent viscosity of $0.10 \mathrm{~d} l / \mathrm{g}$, indicating very low molecular weight. If the same sample of styrene was polymerized with the same amount of AIBN initiator in bulk, polystyrene was obtained with an inherent viscosity of $0.94 \mathrm{~d} l / g$. This indicated that the growing polystyrene radicals most likely had undergone extensive chain transfer with chloral. This is particularly important since it would have been expected that the polymerization of styrene in a rigid matrix might have been subject to the Trommsdorff effect, which would have caused an increase in molecular weight. The molecular weight of polystyrene extracted from the polychloral-polystyrene blends was significantly less than that of the polystyrene obtained under bulk polymerization conditions; this indicates extensive chain transfer, which is expected from the polystyrene radical with chloral. Polystyrene extracted from the polychloralpolystyrene blends was also investigated by PMR spectroscopy and compared with bulkpolymerized polystyrene. The PMR spectra of the two samples were found to be superimposable.

Elemental analysis of polystyrene prepared in the presence of chloral by sequential polymeri- 


\section{S. Corley, P. Kubisa, and O. Vogl}

zation showed $0.78 \%$ of chlorine, indicating a number-average molecular weight of 13,000 or a $\overline{D P}$ of about 100 if one assumes $3 \mathrm{C} 1$ atoms per polymer molecule, a result which is in agreement with the chain transfer activity of the chloral in styrene polymerization. The polystyrene samples on which the viscosity measurements and chlorine analyses were performed had been obtained by methylene chloride extraction from blends which had previously been extracted with acetone, which had removed about $65 \%$ of the polystyrene. Hence the average molecular weight of the total polystyrene produced in this system was undoubtedly lower.

The low molecular weight of polystyrene produced in the sequential polymerization of polychloral and polystyrene is probably the major reason for the difference in observed styrene conversions as determined after methanol and acetone extraction. Much of the polystyrene produced is of sufficiently low molecular weight to be even soluble in, and consequently extracted by, methanol.

Polystyrene was quantitatively extracted by methylene chloride from the blends, as shown by the absence of any of the characteristic polystyrene bands in the infrared spectra of polychloral-polystyrene blends extracted with methylene chloride.

Several of the blend cylinders after extraction with acetone were almost transparent, similar to copolymers of chloral with isocyanates and in sharp contrast to unmodified chloral homopolymer which is nearly opaque. This effect was particularly pronounced with the plugs which contained 10 to $20 \mathrm{wt} \%$ of polystyrene after acetone extraction. Methanol extracted blends did not show this transparency.

Polychloral-Poly(methyl methacrylate) Blends

Blends of polychloral and PMMA were made which contained up to $40 \mathrm{wt} \%$ (up to $50 \mathrm{~mol} \%$ ) of MMA in the original monomer mixture. The chloral was initially polymerized by cryotachensic polymerization with $0.2-2.0-\mathrm{mol} \%$ $\mathrm{Ph}_{3} \mathrm{P}$ as the initiator. As with the chloralstyrene mixtures, the amount of initiator had to be increased as the percentage of MMA in the monomer mixture was increased.

After the sequential polymerization to prepare the blends was completed, the $12 \times 50-\mathrm{mm}$ cy- lindrical pieces were divided into three parts. The first portion was evacuated overnight at $0.025 \mathrm{mmHg}$; it was found that no weight loss occurred, indicating that the polymerization had gone to completion or that the small amount of chloral which may have been left over after the polymerization remained absorbed in the polymer sample. The second portion of the polymer was extracted with methanol to remove monomers and low molecular weight materials and at the same time to degrade unstable polychloral portions. The third portion was extracted with acetone for this same purpose and then divided into two sections, one of which was then extracted with methylene chloride to remove high molecular weight PMMA. Acetone was found to be ineffective in extracting the high molecular weight polymer.

The total conversion after exhaustive methanol extraction was between $85 \%$ and nearly $100 \%$. This is substantially higher than the $60-70 \%$ which was observed for the polychloral-polystyrene blends. The fact that the conversion of MMA to PMMA is almost uniformly 100\% seems to indicate that all the weight loss that is observed during the extraction comes from the degradation of an unstable fraction of polychloral which is, however, much less in amount than in the case of blends prepared from chloral and styrene. These results indicate that the MMA and/or PMMA play an additional beneficial role for the stabilization of the polymer of the chloral portion of the blend and possibly act as acylating agents of living or dormant polychloral chains. This would explain a higher percentage of stable polychloral after extraction the presence of a small amount of carbonyl absorption in polychloral after extraction, and also the fact that only $95 \%$ of PMMA can be extracted. If alkylation of anionic living polychloral with PMMA occurred this would mean the formation of a small amount of PMMA with a polychloral long branch, linked through the ester group. A similar termination reaction has been observed earlier with acid chlorides in anionic chloral polymerization but not yet with esters. ${ }^{9}$

The ratio of polychloral to PMMA in the final polymer is very similar to the initial feed ratio of chloral and MMA. However, at high percentages of MMA the polychloral fraction 
Haloaldehyde Polymers. V.

in the blend is less because of the formation of a higher percentage of an unstable fraction of polychloral which is degraded during the extraction.

PMMA, when extracted from the blends with methylene chloride, showed an inherent viscosity of $0.48 \mathrm{~d} l / \mathrm{g}$, which is comparable to the inherent viscosity $(0.31 \mathrm{~d} l / \mathrm{g})$ of PMMA obtained with the same monomer, initiator concentration, and polymerization temperature in bulk. The extracted sample of PMMA was compared with the bulk-polymerized sample by PMR. The spectra of the two samples were found to be superimposable.

PMMA was extracted to more than $95 \%$ from the blends by methylene chloride (Table II) as shown by the carbon/hydrogen analysis of a sample containing 49 wt $\%$ PMMA before extraction. Likewise, films of polychloral-PMMA blends continued to show definite, though not strong, PMMA carbonyl bands after methylene chloride extraction. It is believed that the lack of total extractability of PMMA is due simply to impaired diffusion of high molecular weight PMMA through the polychloral matrix. It is not believed that grafting occurred, in part because extracted PMMA gave a negative Beilstein test for chlorine and no chlorine was detected by elemental analysis.

Polychloral-Poly(methyl acrylate) (PMA) Blends

Blends of polychloral and PMA were also prepared under conditions similar to those developed for the preparation of polychloralPMMA blends (Table III). The combined monomer conversion to polymer blend after vacuum drying was always greater than $85 \%$, indicating high conversions of both monomers to polymer. The total conversion to polymer blend after methanol extraction decreased as the mole percent MA in the monomer feed mixture increased. The percent conversion of MA to methanol-insoluble PMA dropped from nearly quantitative in the $17-\mathrm{mol} \%$ mixture to $78 \%$ in the mixture containing 50-mol $\%$ MA. The chloral conversion to methanol-stable polychloral decreased from $79 \%$ at $17 \mathrm{~mol} \%$ to $24 \%$ at 50 -mol $\%$ MA in the initial monomer mixture.

These values are also reflected in the drop of the total conversion after methanol extraction from $83 \%$ to $44 \%$ witn increasing MA fraction in the monomer feed. The relatively high value of MA conversion to PMA combined with a low chloral conversion value gave a final blend mixture consisting of 2 parts of PMA to 1 part of polychloral at 50-mol $\%$ MA in the feed.

In polychloral-PMA blends the extracted stable blends are enriched in PMA over the initial monomer feed ratios and indicate that an unusually large proportion of the polychloral portion of the blend is degraded during the methanol extraction. We have no plausible explanation at this time why a much larger fraction of the chloral polymer portion is unstable $(76 \% v s .32 \%$ in the standard) when the chloral polymerization with $\mathrm{Ph}_{3} \mathrm{P}$ is carried out in the presence of large amounts of MA. A larger amount of $\mathrm{Ph}_{3} \mathrm{P}$ initiator had always been used for the chloral polymerization at the higher concentrations of olefinic monomers used for the blends, but no such pronounced decrease of the stable fraction had been detected in blends of polychloral-polystyrene and polychloralPMMA at higher concentrations of $\mathrm{Ph}_{3} \mathrm{P}$ and olefinic addition monomer.

The acetone extracted samples of high PMApolychloral ratio also show substantially decreased conversion of chloral to polychloral, which can only mean that this is an inherent property of the sample, not of the extraction method.

PMA extracted from the blends of polychloral and PMA had an inherent viscosity of $0.31 \mathrm{~d} l / \mathrm{g}$, in comparison with an inherent viscosity of $0.89 \mathrm{~d} l / g$ for PMA prepared in bulk with the same monomer, initiator concentration, and polymerization temperature as for the blend polymerization. It could be that in the bulk polymerization, branching occurred with a subsequently higher viscosity-average molecular weight in the resultant polymer, which is not possible in the case of the MMA polymerization, but we have not attempted to establish this fact.

Some chain transfer with chloral monomer does occur in the radical polymerization of MA in the polychloral matrix, because acetone-extracted PMA showed a chlorine content of $0.69 \%$ by elemental analysis. With the assumption of $3 \mathrm{C} 1$ atoms per polymer molecule, this result indicates a number-average molecular weight of 15,000 , corresponding to a $\overline{D P}$ of about 180. This chain transfer in MA polymeri- 
zation is not as effective as in styrene polymerization but more effective than in MMA polymerization, which showed no incorporation of chlorine into PMMA and whose average molecular weight was also not decreased when the polymerization was carried out in sequence after chloral polymerization.

\section{Polychloral-Crosslinked Polystyrene Interpenetrat-} ing Networks

Sequential polymerization of chloral and styrene was also carried out in the presence of $5.4 \%$ of divinylbenzene to crosslink polystyrene and to form an interpenetrating network of polychloral with crosslinked polystyrene (Table IV). As can be seen, the total conversion of monomers to polymers is higher than $90 \%$ after vacuum drying at $0.025 \mathrm{mmHg}$.

The second part of the polymer sample was then extracted for $48 \mathrm{hr}$ with refluxing benzene. The benzene extracted unreacted monomers (chloral, styrene, and divinylbenzene) and also the unstable portion of polychloral and extracted a noncrosslinked portion of polystyrene. In a separate experiment it was shown by IR spectroscopy that the extraction with refluxing benzene was effective in removing the noncrosslinked polystyrene from the polychloral matrix.

As shown in Table IV, the total conversion of the monomer mixture to the IPN was relatively low, though highest for low percentages of styrene in the monomer feed. It is known that as much as $10 \%$ of unreacted chloral monomer and $20-25 \%$ of unstable polychloral may be present in the portion of the polymerization mixture that comes from the chloral part of the monomer feed which is degraded and extracted by benzene. In general, half of the polystyrene that is produced in the polychloral matrix is crosslinked and the other half can be extracted by benzene.

\section{Polychloral-Crosslinked Poly(methyl methacry-} late) Interpenetrating Networks

Interpenetrating networks of polychloral and PMMA crosslinked with 7.6-wt $\%$ ethylene dimethacrylate (EDMA) were prepared as films about $0.03-\mathrm{mm}$ thick by sequential polymerization with $0.4-0.8 \mathrm{~mol} \%$ of $\mathrm{Ph}_{3} \mathrm{P}$ as the initiator for the MMA polymerization.

The total conversion to polymers after drying overnight at room temperature at $0.025 \mathrm{mmHg}$ was more than $95 \%$. Extraction for $48 \mathrm{hr}$ with refluxing benzene removed $25-40 \%$ of the polymer. The relatively low conversion to polymer after benzene extraction, as compared to the methanol extraction of noncrosslinked PMMA in polychloral (Table II), indicates that the $20 \%$ difference in the extracted total yield of the polymers is probably caused by degrative extraction of polychloral which seems to occur more effectively with benzene but not with methanol. Poly(methyl methacrylate) in the IPN seems to be completely crosslinked at medium or high concentrations of MMA but not at low concentrations of MMA. In IPN's from chloral and MMA, crosslinked with EDMA, the polychloral part still has a very substantial unstable fraction, which is most noticeable in IPN's with high MMA (and EDMA) content.

Further work on the preparation of polymer blends and IPN's based on chloral homo- and copolymers with olefinic addition polymers will be necessary to describe in greater detail the chemical composition, morphological structure, and physical and mechanical structures of these polymer composites. These characterizations are very important because the reproducibility of these preparations depends on many factors which have to be observed very carefully.

Acknowledgements. This work was supported by the National Science Foundation and the Materials Research Laboratory of the University of Massachusetts. One of us (P. K.) was on a leave of absence from the Centre of Molecular and Macromolecular Studies in Lodz, Poland, to work at the University of Massachusetts.

We would also like to express our thanks to the Montrose Chemical Company, particularly Mr. H. J. Wurzer, for their generous supply of chloral.

\section{REFERENCES}

1. O. Vogl, H. C. Miller, and W. H. Sharkey, Macromolecules, 5, 658 (1972).

2. O. Vogl, U.S. Patent 3454527 (1969).

3. O. Vogl, U. S. Patent 3707524 (1972).

4. K. Hatada, L. S. Corley, Sh. S. Vezirov, and O. Vog1, Vysokomol. Soedin., in press.

5. O. Vogl, U. S. Patent 3668184 (1972). 


\section{Haloaldehyde Polymers. V.}

6. O. Vogl, U.S. Patent 3775371 (1973).

7. S. C. Kim, D. Klempner, K. S. Frisch, W. Radigan, and H. L. Frisch, Macromolecules, 9, 258 (1976).
8. P. Kubisa and O. Vog1, Macromol. Synth., in press.

9. P. Kubisa and O. Vog1, Polym. J., 7, 186 (1975). 